\title{
Performance Comparison between Nonidentical Segmented Exponential Concave and Nonidentical Segmented Exponential Convex Serrated CATRs
}

\author{
T. Venkata Rama Krishna, ${ }^{1}$ P. Siddaiah, ${ }^{1}$ and B. Prabhakara Rao ${ }^{2}$ \\ ${ }^{1}$ Koneru Lakshmaiah College of Engineering, Vaddeswaram, Guntur District 522-501, \\ Andhra Pradesh, India \\ ${ }^{2}$ College of Engineering, Jawaharlal Nehru Technological University (JNTU), Kakinada 533-033, \\ Andhra Pradesh, India \\ Correspondence should be addressed to T. Venkata Rama Krishna, tottempudi_rk@yahoo.com
}

Received 1 June 2007; Accepted 27 November 2007

Recommended by Charles Bunting

\begin{abstract}
This paper presented a theoretical and numerical assessment for nonidentical segmented exponential- (NISE-) convex and NISEconcave serrated plane CATRs by changing number of serrations. The investigation was based on diffraction theory and, more specifically, on the diffraction formulation of Fresnel. The compact antenna test range (CATR) provides uniform illumination within the Fresnel region to the test antenna. Application of serrated edges has been shown to be a good method to control diffraction at the edges of the reflectors. In this paper, the Fresnel fields of NISE-convex and NISE-concave serrated CATRs are analyzed using physical optics (PO) technique. The PO analysis is applied in this paper for plane reflector serrated CATR only. The same analysis is applied to any type of reflector. In this paper, lens-based reflector is not considered. It is observed that NISE-concave serrated CATR gives less ripple and enhanced quiet zone width than NISE-convex.
\end{abstract}

Copyright (c) 2008 T. Venkata Rama Krishna et al. This is an open access article distributed under the Creative Commons Attribution License, which permits unrestricted use, distribution, and reproduction in any medium, provided the original work is properly cited.

\section{INTRODUCTION}

The CATR provides a relatively new method of measuring the far-field characteristics of microwave antennas. The test antenna is illuminated by a collimated beam of energy from a paraboloid. The linear dimensions of the reflector must be at least three to four times that of the test antenna in order that the illumination on it approximates a plane wave. This technique leads to far field pattern simulation and requires smaller distances than in conventional methods. A CATR can be situated in a normal anechoic chamber. Due to the good indoor performance, the method is time- and cost-effective and the measurements are performed in real time. Rigorous analysis of the CATR is needed in order to obtain a realistic assessment of the test zone fields. Some factors which should be taken into account are as follows:

(i) nonideal system components, such as tapered feed pattern and mechanical inaccuracies of the reflectors; (ii) nonideal environment as due to reflections from walls and obstacles in the anechoic room;

(iii) stray radiation level;

(iv) reflector surface accuracy; and

(v) edge diffraction at the CATR reflectors that contribute to scattering at the edges of the reflector, thus modifying the far-field pattern.

The fifth factor has a large impact on the performance of the test-zone field. The methods to reduce edge diffraction are as follows.

(i) One method is creating an incident field with an amplitude pattern that falls off sharply at angles corresponding to the edges of the reflector. Such a feed pattern is not easy to create over a large range of frequencies and it reduces effective test-zone size.

(ii) Another method is to use of microwave absorber at the reflector edges. 


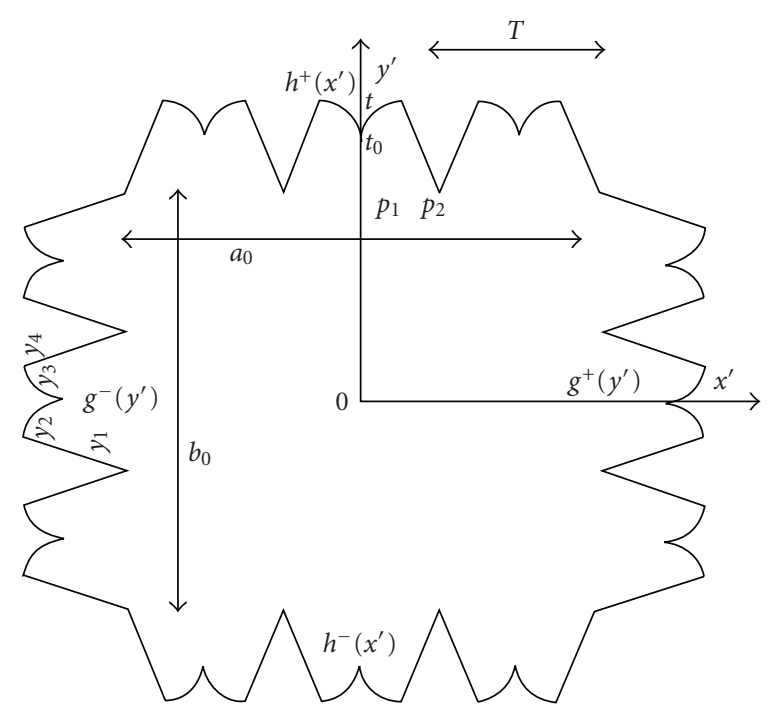

FIGURE 1: Square aperture reflector with NISE-convex serrations.

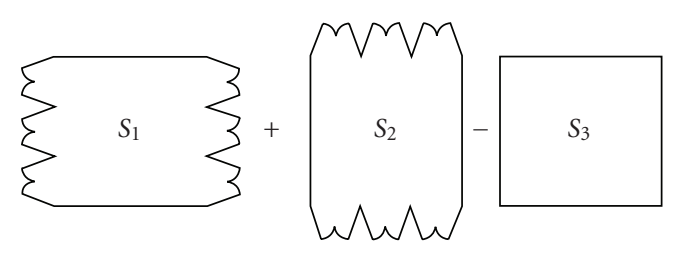

Figure 2: Decomposition of aperture $S$ into three parts $S_{1}, S_{2}$, and $S_{3}$.

(iii) The third method is to use of small flanges around the aperture edges.

(iv) The fourth method depends on serrating the edges of the reflector in order to redistribute the diffracted rays in many directions away from the quiet zone area.

Application of serrated edges is a good method of controlling the diffraction effects at the edges of the reflector. The way in which the reflector surface is terminated affects the electromagnetic properties of the radiating system. The signal reflected by the parabolic reflector is basically a plane wave which exists over the extent of the surface. This reflected field abruptly stops at the surface termination. Since the electromagnetic fields must be smooth and continuous, there will be strong diffracted field emanating from the terminating edges. This field then interferes constructively and destructively with the plane wave causing amplitude and phase variations of the field illuminating the test antenna or scattering body and degrades the performance of the range. This diffracted signal is one of the major contributions limiting the use of the compact ranges.

The serrated edge termination is intended to form a transition between the reflector surface and free space. In performing this function, it is designed to diffract energy away from the target zone. However, a finite edge is composed of corners which diffract energy in all directions including the target zone. The use of serrated edges is tantamount to creating corners which can also diffract into the target zone. Each

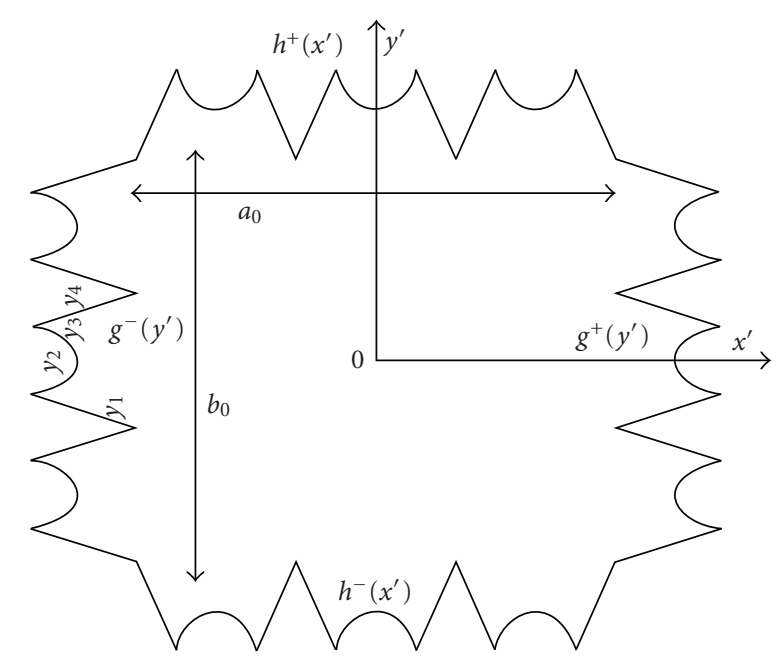

FIGURE 3: Square aperture reflector with NISE-concave serrations.

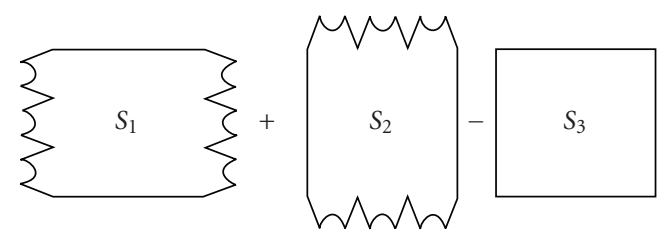

FIgURE 4: Decomposition of aperture $S$ into three parts $S_{1}, S_{2}$, and $S_{3}$.

of these corner diffracted fields will be relatively weak. Appropriate designs of serrations ensure effective cancellation of these diffraction components in the test (quiet) zone. It has been shown theoretically that the application of serrations minimizes distorting diffraction effects in the Fresnel region of a CATR $[1,2]$.

\section{EVALUATION OF FRESNEL FIELD OF SERRATED EDGE REFLECTOR BY PO ANALYSIS}

The analysis of the Fresnel field of a square aperture with NISE serrations is applied using the method of PO. In this paper, four different shapes of serrations are used in four sides. This analysis is so general that it can be applied to any serration geometry. This paper presents a gist of the analysis of NISE-convex and NISE-concave geometries shown in Figures 1 and 3. The Fresnel diffraction formula which gives the $\mathrm{x}$-polarized field over an arbitrary plane $(z=$ constant $)$ in the Fresnel region is $[3,4]$

$$
\begin{aligned}
E_{x}(x, y, z)= & -\frac{j k}{2 \pi z} e^{-j k z} \int_{-\infty}^{\infty} \int_{-\infty}^{\infty} E_{a x}\left(x^{\prime}, y^{\prime}\right) \\
& \times e^{j k\left\{\left(x^{\prime}-x\right)^{2}+\left(y^{\prime}-y\right)^{2}\right\} / 2 z} d x^{\prime} d y^{\prime} .
\end{aligned}
$$

It will be a laborious task to find an analytical expression in a closed form for the Fresnel diffraction pattern of an aperture with these serrated edges. Hence, recourse is taken to decompose the aperture area $S$ into three parts $S_{1}, S_{2}$, and $S_{3}$, such that $S=S_{1}+S_{2}-S_{3}$ (see, Figures 2 and 4 ). A quasianalytical 
expression can now be derived for the Fresnel field [5-9]. The NISE-convex and NISE-concave serrations described by the boundary functions $h^{+}\left(x^{\prime}\right), h^{-}\left(x^{\prime}\right)$ and $g^{+}\left(y^{\prime}\right), g^{-}\left(y^{\prime}\right)$ are expressed as Fourier series of width-modulated exponential with rate of rise " $a_{i}$." The serrated edges are described by the functions $h^{+}\left(x^{\prime}\right), h^{-}\left(x^{\prime}\right)$ and $g^{+}\left(y^{\prime}\right), g^{-}\left(y^{\prime}\right)$, and $E_{a x}\left(x^{\prime}, y^{\prime}\right)=E_{0}$ for $\left(x^{\prime}, y^{\prime}\right) \in S$.

Now, (1) can be rearranged as

$$
E_{x}(x, y, z)=\frac{-j E_{0}}{2} e^{-j k z}\left(I_{1}+I_{2}+I_{3}\right)
$$

where

$$
\begin{aligned}
& I_{1}=\frac{k}{\pi z} \int_{-h=-b_{0} / 2}^{h=b_{0} / 2} e^{j k\left(y^{\prime}-y\right)^{2} / 2 z} d y^{\prime} \int_{g^{-}\left(y^{\prime}\right)}^{g^{+}\left(y^{\prime}\right)} e^{j k\left(x^{\prime}-x\right)^{2} / 2 z} d x^{\prime}, \\
& =\frac{k}{\pi z}\left[F\left(t_{+}\right)-F\left(t_{-}\right)\right]\left[F\left(s_{+}^{\prime}\right)-F\left(s_{-}^{\prime}\right)\right] ; \\
& I_{2}=\frac{k}{\pi z} \int_{-w=-a_{0} / 2}^{w=a_{0} / 2} e^{j k\left(x^{\prime}-x\right)^{2} / 2 z} d x^{\prime} \int_{h^{-}\left(x^{\prime}\right)}^{h^{+}\left(x^{\prime}\right)} e^{j k\left(y^{\prime}-y\right)^{2} / 2 z} d y^{\prime}, \\
& =\frac{k}{\pi z}\left[F\left(s_{+}\right)-F\left(s_{-}\right)\right]\left[F\left(t_{+}^{\prime}\right)-F\left(t_{-}^{\prime}\right)\right] ; \\
& I_{3}=\frac{k}{\pi z} \int_{-w=-a_{0} / 2}^{w=a_{0} / 2} e^{j k\left(x^{\prime}-x\right)^{2} / 2 z} d x^{\prime} \int_{-h=-b_{0} / 2}^{h=b_{0} / 2} e^{j k\left(y^{\prime}-y\right)^{2} / 2 z} d y^{\prime}, \\
& =\frac{k}{\pi z}\left[F\left(s_{+}\right)-F\left(s_{-}\right)\right]\left[F\left(t_{+}\right)-F\left(t_{-}\right)\right] ; \\
& t_{ \pm}=\sqrt{\frac{k}{\pi z}}( \pm h-y) \text {; } \\
& s_{+}^{\prime}=\sqrt{\frac{k}{\pi z}}\left(-g^{-}\left(y^{\prime}\right)-x\right) \text {; } \\
& s_{-}^{\prime}=\sqrt{\frac{k}{\pi z}}\left(-g^{+}\left(y^{\prime}\right)-x\right) \text {; } \\
& s_{ \pm}=\sqrt{\frac{k}{\pi z}}( \pm w-x) \\
& t_{+}^{\prime}=\sqrt{\frac{k}{\pi z}}\left(-h^{-}\left(x^{\prime}\right)-y\right) ; \\
& t_{-}^{\prime}=\sqrt{\frac{k}{\pi z}}\left(-h^{+}\left(x^{\prime}\right)-y\right) ; \\
& F(s)=\int_{0}^{s} e^{-j \pi r^{2} / 2} d r \\
& =\text { the complex form of the Fresenel integral. }
\end{aligned}
$$

\subsection{Fourier series of width- and height-modulated NISE-convex serrations}

The serrations described by the boundary functions $h^{+}\left(x^{\prime}\right), h^{-}\left(x^{\prime}\right)$ and $g^{+}\left(y^{\prime}\right), g^{-}\left(y^{\prime}\right)$ are expressed as a Fourier series of width- and height-modulated nonidentical

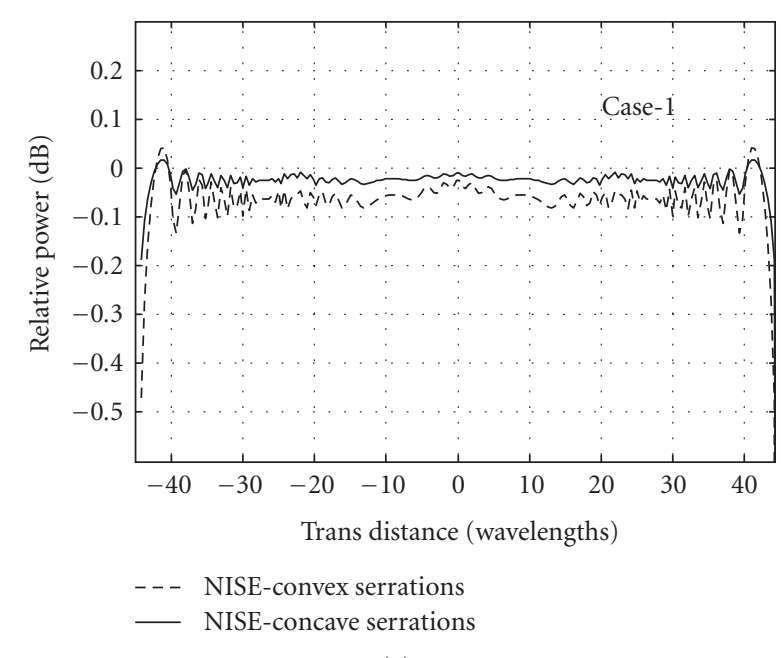

(a)

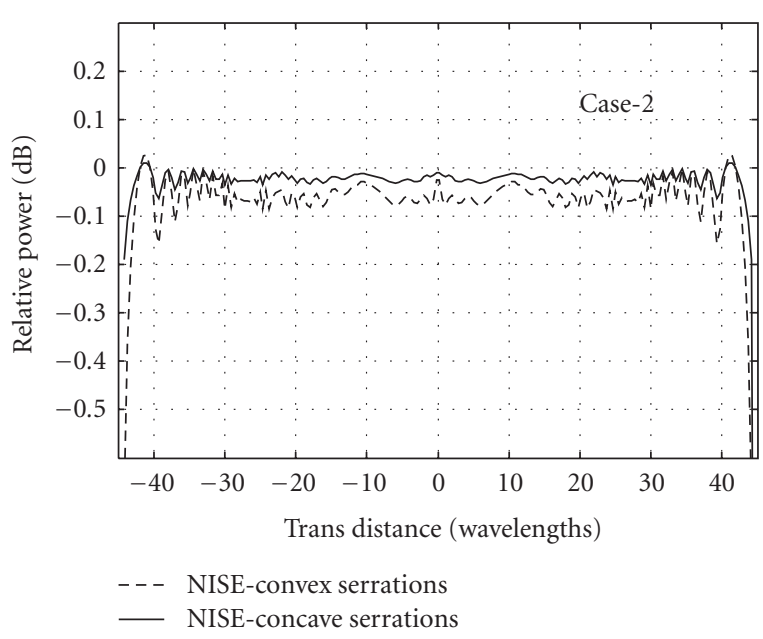

(b)

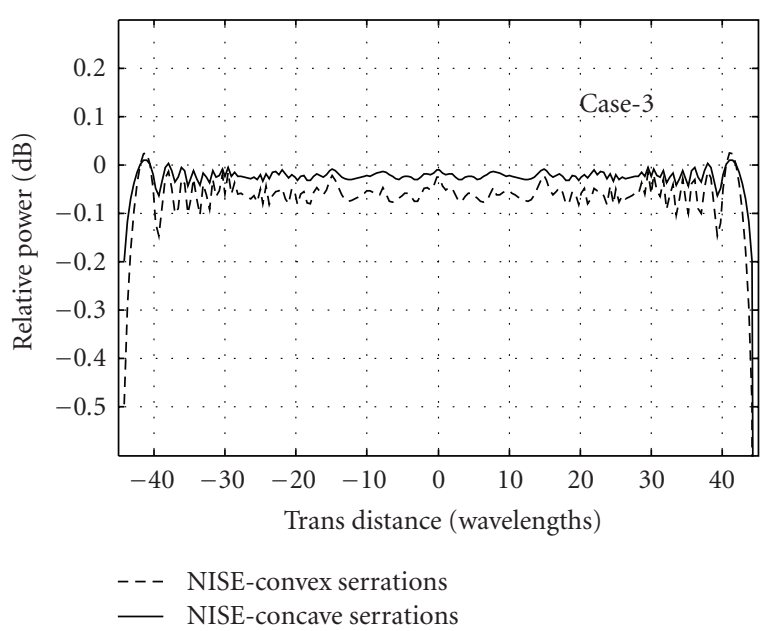

(c)

Figure 5: Fresnel-zone field for $90 \lambda \times 90 \lambda$ NISE-convex and NISEconcave serrated CATRs for cases 1,2 , and 3 . 
TABLE 1: Nonidentical exponential serration functions.

\begin{tabular}{lll}
\hline & Nonidentical segmented concave & Nonidentical segmented convex \\
\hline & $f\left(x^{\prime}\right)=y_{1}+y_{2}+y_{3}+y_{4}$, & $f\left(x^{\prime}\right)=y_{1}+y_{2}+y_{3}+y_{4}$, \\
& $y_{1}=\frac{t}{p_{2}-p_{1}}\left(x^{\prime}+p_{2}\right), \quad-p_{2}<x^{\prime}<-p_{1}$, & $y_{1}=\frac{t}{p_{2}-p_{1}}\left(x^{\prime}+p_{2}\right), \quad-p_{2}<x^{\prime}<-p_{1}$, \\
Defining equation & $y_{2}=t_{0} e^{-a x^{\prime}}, \quad-p_{1}<x^{\prime}<0$, & $y_{2}=t_{0}+t\left(1-e^{a x^{\prime}}\right), \quad-p_{1}<x^{\prime}<0$ \\
& $y_{3}=t_{0} e^{a x^{\prime}}, \quad 0<x^{\prime}<p_{1}$, & $y_{3}=t_{0}+t\left(1-e^{-a x^{\prime}}\right), \quad 0<x^{\prime}<p_{1}, \quad p_{4}$ \\
& $y_{4}=\frac{t}{p_{1}-p_{2}}\left(x^{\prime}-p_{2}\right), \quad p_{1}<x^{\prime}<p_{2}$, & $y_{4}=\frac{t}{p_{1}-p_{2}}\left(x^{\prime}-p_{2}\right), \quad p_{1}<x^{\prime}<p_{2}, \quad$ \\
& where $t_{0}=t e^{-a p_{1}}$. & where $t_{0}=t e^{-a p_{1} .}$.
\end{tabular}

TABLE 2: Width modulation factors for NISE serrations.

\begin{tabular}{lcccc}
\hline CASE & $P$ & $P_{1} / P$ & $P_{2} / P$ & Number of serrations \\
\hline 1 & $\left(a_{0} / 2\right) / 112.5$ & 6.0 & 45 & 1 \\
2 & $\left(a_{0} / 2\right) / 56.25$ & 5.333 & 22.50 & 2 \\
3 & $\left(a_{0} / 2\right) / 37.50$ & 4.667 & 15.0 & 3 \\
4 & $\left(a_{0} / 2\right) / 28.125$ & 4.0 & 11.25 & 4 \\
5 & $\left(a_{0} / 2\right) / 22.50$ & 1.5 & 9 & 5 \\
6 & $\left(a_{0} / 2\right) / 18.75$ & 3.0 & 7.50 & 7 \\
7 & $\left(a_{0} / 2\right) / 16.07$ & 2.66 & 6.428 & 8 \\
8 & $\left(a_{0} / 2\right) / 14.0625$ & 2.0 & 5.625 & 9 \\
9 & $\left(a_{0} / 2\right) / 12.50$ & 1.333 & 5.0 & \\
\hline
\end{tabular}

TABLE 3: Height modulation factor for NISE serrations.

$$
\frac{\bar{t}}{1 \lambda}
$$

segmented convex function. The Fourier series expansion of $h^{+}\left(x^{\prime}\right), h^{-}\left(x^{\prime}\right)$ and $g^{+}\left(y^{\prime}\right), g^{-}\left(y^{\prime}\right)$ is given by

$$
\begin{aligned}
& h^{+}\left(x^{\prime}\right)= h^{-}\left(x^{\prime}\right)=g^{+}\left(x^{\prime}\right)=g^{-}\left(x^{\prime}\right)=C_{01}+\sum_{n=-\infty}^{\infty} C_{n 1} e^{(j n \omega / T) t}, \\
& C_{01}=\frac{1}{T}\left[2 p_{1}\left(t_{0}+t\right)+\frac{t}{a}\left(1-e^{-a p_{1}}\right)+t\left(p_{2}-p_{1}\right)\right], \\
& C_{n 1}=\frac{1}{T}\left[-\frac{2 t a}{a^{2}+n^{2} \omega^{2}}+\frac{2\left(t_{0}+t\right)}{n \omega} \sin \left(n \omega p_{1}\right)\right. \\
&+\frac{2 t_{1}}{\left(p_{2}-p_{1}\right)}\left(\frac{p_{1}}{n \omega} \sin \left(n \omega p_{1}\right)+\frac{1}{n^{2} \omega^{2}}\right. \\
& \times\left(\cos \left(n \omega p_{1}\right)-\cos \left(n \omega p_{2}\right)\right) \\
&+\frac{2 t e^{-a p_{1}}}{a^{2}+n^{2} \omega^{2}}\left(a \cos \left(n \omega p_{1}\right)-n \omega \sin \left(n \omega p_{1}\right)\right)
\end{aligned}
$$

\subsection{Fourier series of width- and height-modulated NISE-concave serrations}

The serrations described by the boundary functions $h^{+}\left(x^{\prime}\right), h^{-}\left(x^{\prime}\right)$ and $g^{+}\left(y^{\prime}\right), g^{-}\left(y^{\prime}\right)$ are expressed as a Fourier series of width and height modulated nonidentical segmented concave function. The Fourier series expansion of $h^{+}\left(x^{\prime}\right), h^{-}\left(x^{\prime}\right)$ and $g^{+}\left(y^{\prime}\right), g^{-}\left(y^{\prime}\right)$ is given by

$$
\begin{aligned}
h^{+}\left(x^{\prime}\right)=h^{-}\left(x^{\prime}\right) & =g^{+}\left(x^{\prime}\right)=g^{-}\left(x^{\prime}\right)=C_{02}+\sum_{n=-\infty}^{\infty} C_{n 2} e^{(j n \omega / T) t}, \\
C_{02}=\frac{1}{T} & \left.t\left(p_{2}-p_{1}\right)+\frac{2 t_{0}}{a}\left(e^{a p_{1}}-1\right)\right], \\
C_{n 2}=\frac{1}{T}[- & \frac{2 t a}{a^{2}+n^{2} \omega^{2}}+\frac{2 t_{1}}{\left(p_{2}-p_{1}\right)} \\
& \times\left(\frac{p_{1}}{n \omega} \sin \left(n \omega p_{1}\right)+\frac{1}{n^{2} \omega^{2}}\right. \\
& \times\left(\cos \left(n \omega p_{1}\right)-\cos \left(n \omega p_{2}\right)\right) \\
& \left.-\frac{p_{2}}{n \omega} \sin \left(n \omega p_{1}\right)\right) \\
+ & \left.\frac{2 t_{0} e^{a p_{1}}}{a^{2}+n^{2} \omega^{2}}\left(a \cos \left(n \omega p_{1}\right)-n \omega \sin \left(n \omega p_{1}\right)\right)\right] .
\end{aligned}
$$




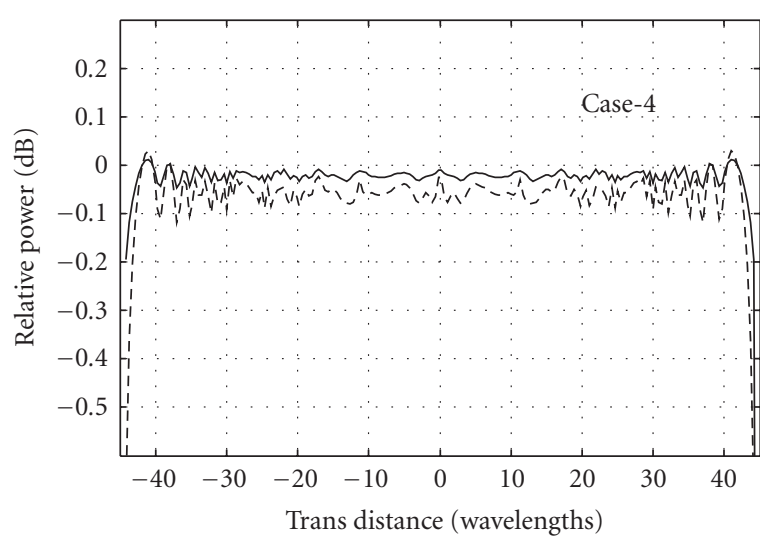

- - - NISE-convex serrations

— NISE-concave serrations

(a)

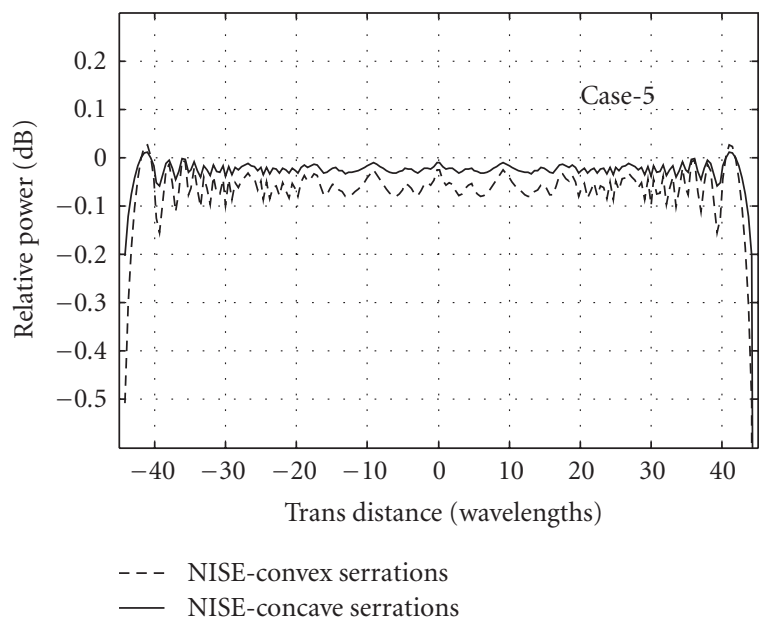

(b)

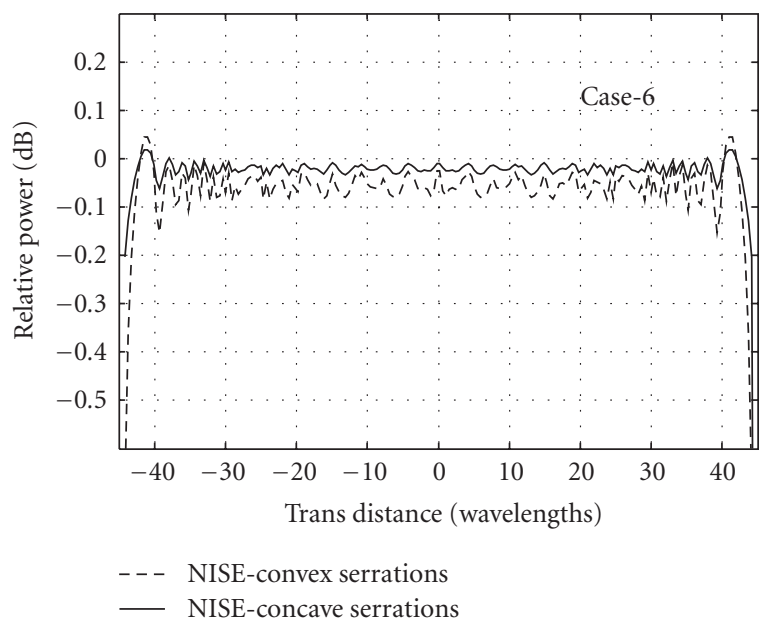

(c)

FIGURE 6: Fresnel-zone field for $90 \lambda \times 90 \lambda$ NISE-convex and NISEconcave serrated CATRs for cases 4,5 , and 6 .

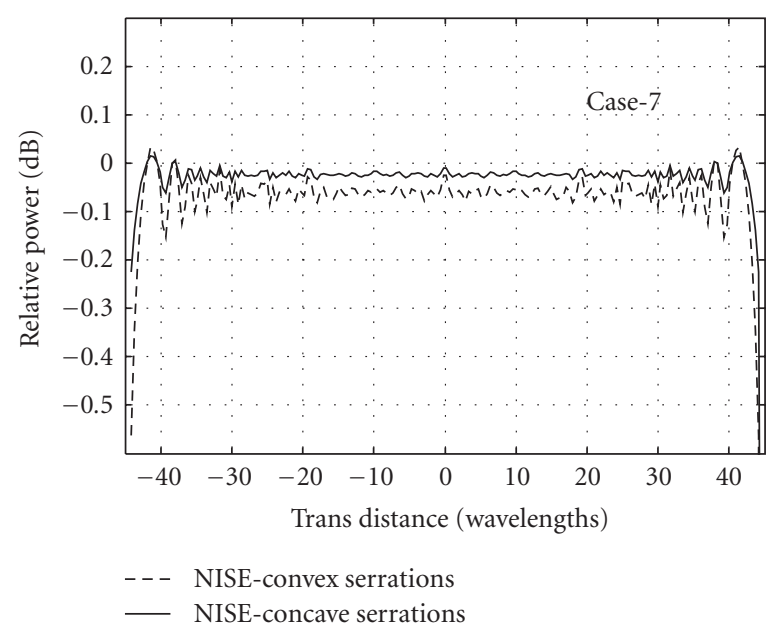

(a)

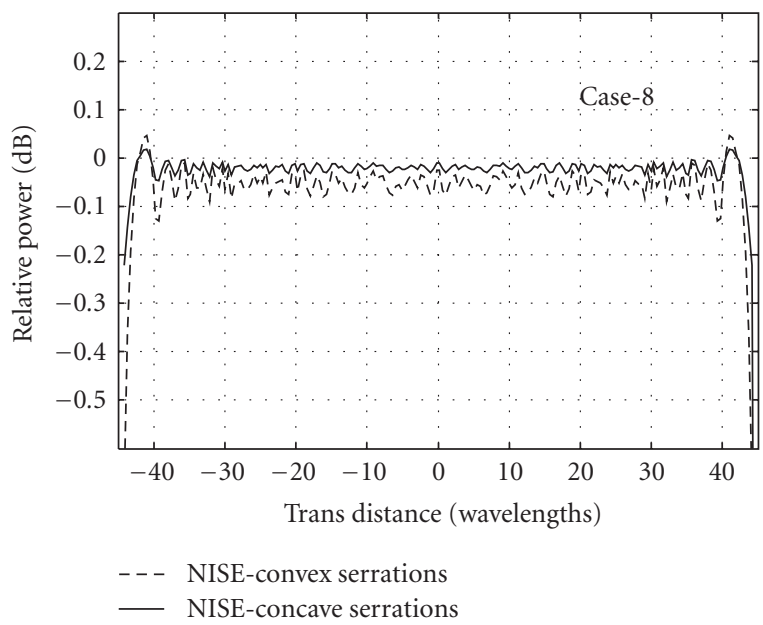

(b)

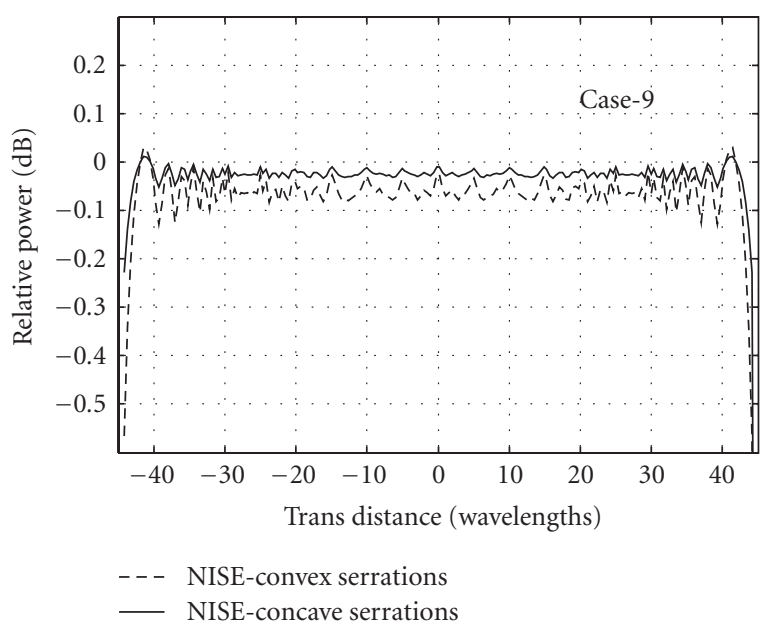

(c)

FIGURE 7: Fresnel-zone field for $90 \lambda \times 90 \lambda$ NISE-convex and NISEconcave serrated CATRs for cases 7,8 , and 9 . 


\section{RESULTS AND DISCUSSION}

The technique presented here is best suited to the analysis of serrated reflectors commonly employed in compact range systems for reduced edge diffraction. A square reflector of aperture dimensions $90 \lambda \times 90 \lambda$ is considered to be equipped with NISE. Equations in Table 1 represent the Fourier series of NISE-concave and NISE-convex serrations. Table 2 represents width-modulation factors and a number of serrations used for CATR. Table 3 represents height of the serrations. Equations in Table 1 have been used in conjunction with (3) to evaluate the Fresnel field at a transverse distance in wavelengths at a distance of $z=64 \lambda$ from the reflector aperture plane over the line $y=0,0<x<90 \lambda$. An integration step size of $0.25 \lambda$ has been used. The Fresnel integral was simulated using Matlab7.01 [10]. The Fresnel field is computed for the different values of width- and height-modulation factors indicated in Tables 2 and 3. The relative power in $\mathrm{dB}$ versus transverse distance in wavelengths with the space constant $a_{i}=0.6$ for exponential serrations is presented for different cases in Figures (5-7). This implies less ripple at the centre of the quiet zone which is indicative of better cancellation of diffraction effects.

\section{CONCLUSIONS}

This paper presented a performance evaluation of the NISEserrated edge reflector with rectangular aperture for different values of width- and height-modulation factors. The quiet zone field of a $90 \lambda \times 90 \lambda$ is assessed for different cases as illustrated in Tables 2 and 3. Quiet zone width and ripple parameters are to be considered as a-figure-of-merit factors. These two factors are the deciding parameters regarding which serrated CATR gives better performance. From Figures 5,6 , and 7 , it is observed that less ripple and enhanced quiet zone width are observed in this NISE-concave serrated CATR than NISE-convex serrated CATR. It is concluded that NISEconcave serrated CATRs give better performance. This NISEserrated edge CATR gives better quiet size and less ripples than triangular and on/off rectangular serrated CATRs.

\section{ACKNOWLEDGMENTS}

The authors place on record their grateful thanks to the authorities of the K L College of Engineering, Guntur, India and Jawaharlal Nehru Technological University, College of Engineering, Kakinada, India for providing the facilities.

\section{REFERENCES}

[1] J. S. Hollis, T. J. Lyon, and L. Clayton, Microwave Antenna Measurements, Scientific Atlanta, Atlanta, Ga, USA, 1985.

[2] G. E. Evans, Antenna Measurements Techniques, Artech House, London, UK, 1990.

[3] K. D. Mielenz, "Algorithms for Fresnel diffraction at rectangular and circular apertures," Journal of Research of the National Institute of Standards and Technology, vol. 103, no. 5, pp. 497509, 1998.

[4] T. V. R. Krishna, P. Siddaiah, and B. P. Rao, "Antenna measurements: a comparison of triangular on-off serrated CATR, trapezoidal on-off serrated CATR techniques," in Proceedings of the 2nd International Conference on (WCSN '06), pp. 345351, Allahabad, India, December 2006.

[5] S. A. Akhmanov and S. Y. Nikitin, Physical Optics, Clarendon Press, Oxford Press, New York, NY, USA, 1997.

[6] M. Abramowitz and I. A. Stegun, Eds., Handbook of mathematical functions, National Bureau of Standards, Washington, DC, USA, 1964.

[7] P. Siddaiah, P. V. Subbaiah, and T. V. R. Krishna, "Performance comparison between HMCE \& IMCE serrated CATRs," in Topics in Electromagnetic Waves Devices, Effects and Applications, pp. 13-20, Anamaya, New Delhi, India, 2005.

[8] T. V. R. Krishna, P. Siddaiah, and B. P. Rao, "Performance analysis of CATR reflector with super hybrid modulated segmented exponential serrated edges," International Journal of Electrical, Computer, and Systems Engineering, vol. 1, no. 3, pp. 177-182, 2007.

[9] B. P. Rao, P. Siddaiah, and T. V. R. Krishna, "Performance comparison between hybrid and super hybrid serrated CATRs," in Proceedings of the 29th Annual Symposium of the Antenna Measurement Techniques Association (AMTA '07), St. Louis, Mo, USA, November 2007.

[10] W. Gander and W. Gautschi, "Adaptive quadraturerevisited," BIT Numerical Mathematics, vol. 40, no. 1, pp. 84 $101,2000$. 

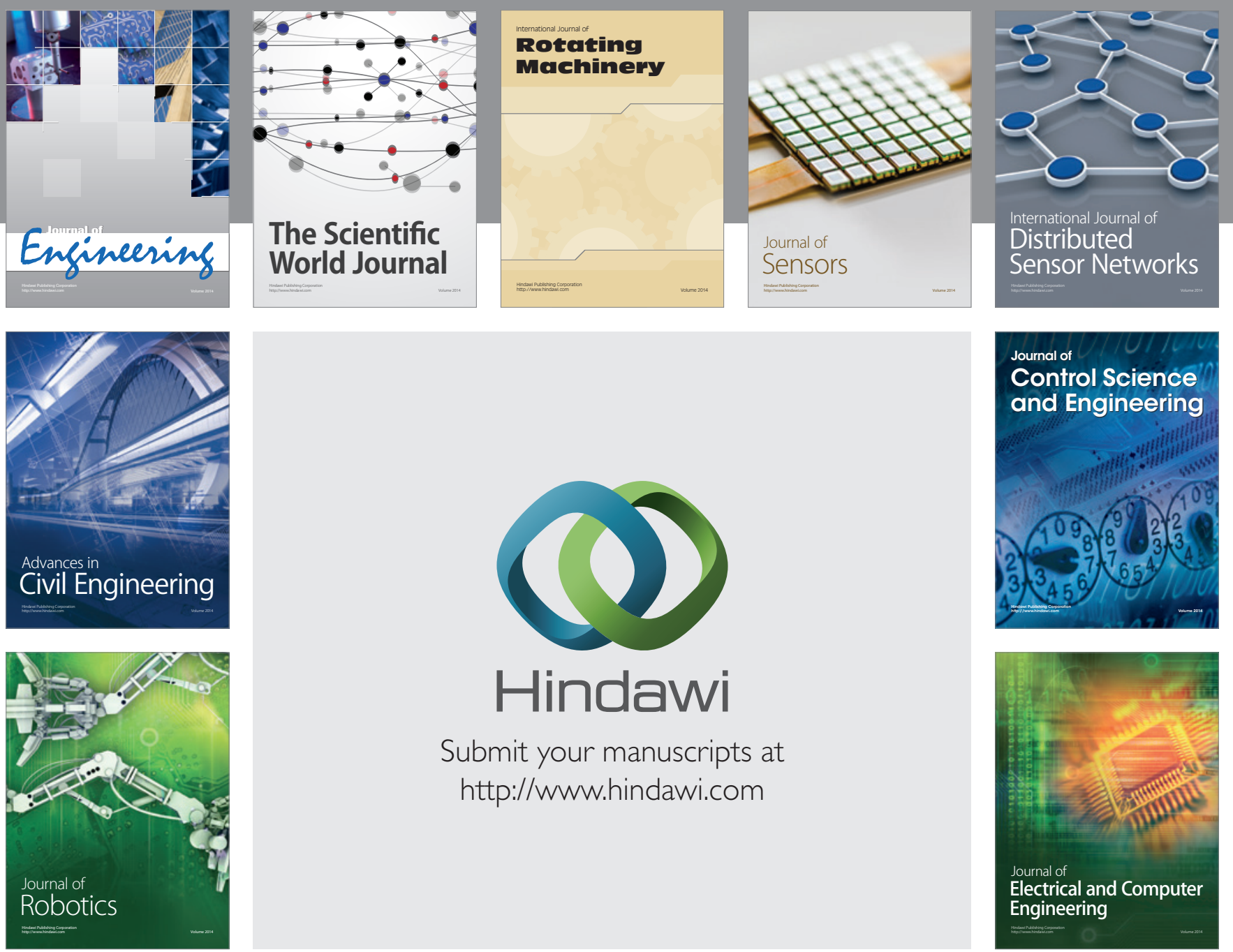

Submit your manuscripts at

http://www.hindawi.com
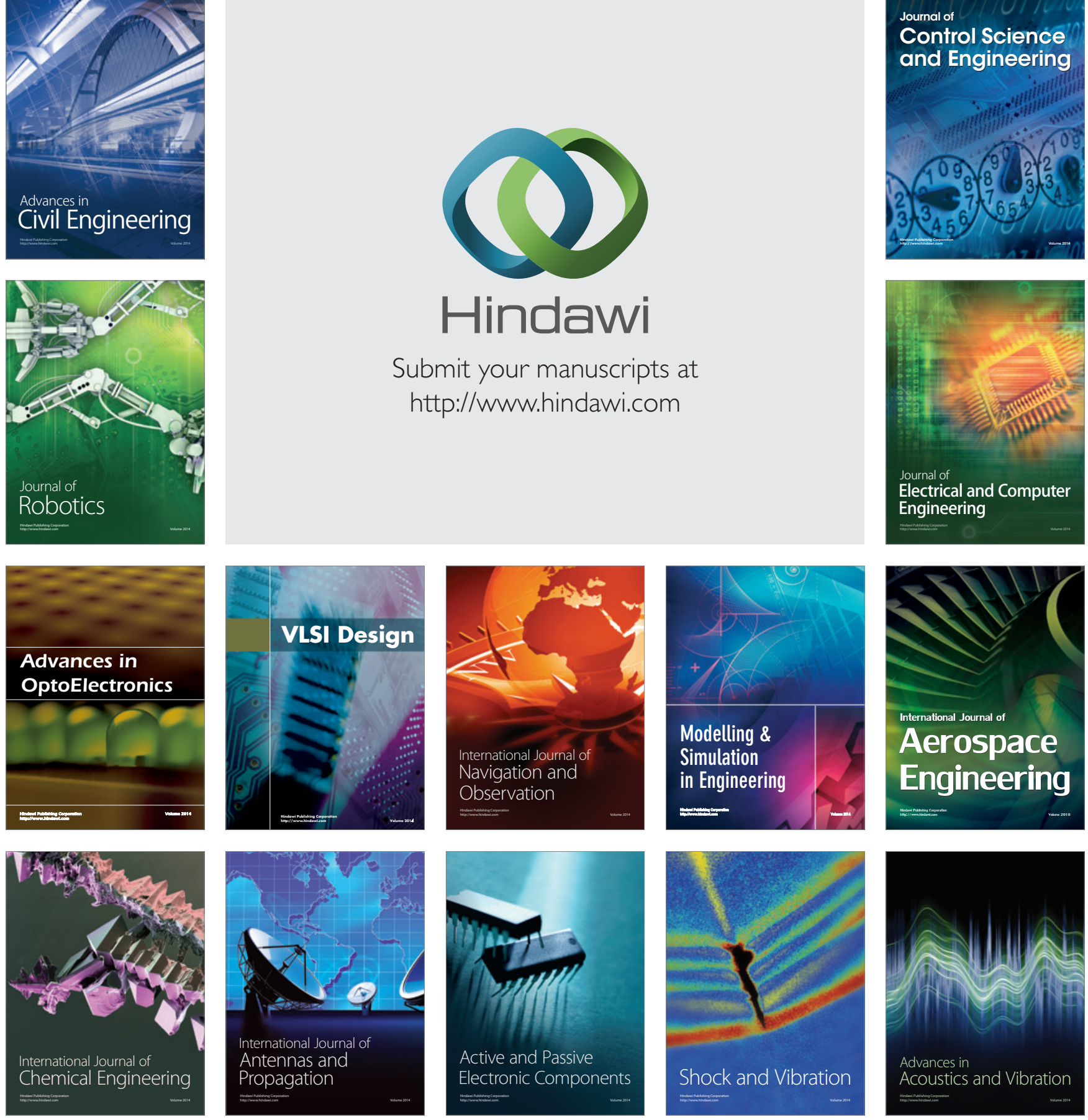